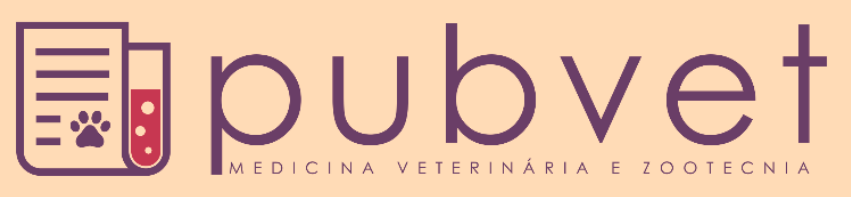

https://doi.org/10.31533/pubvet.v13n12a461.1-5

\title{
Prevalência de contaminação fúngica em rações vendidas a granel na cidade de Teresina, Piauí
}

\author{
Laide Danielle Coelho da Silva Chaves ${ }^{1 *} \bullet$, Artur Oliveira Rocha ${ }^{1}$, Wanderson Gabriel Gomes \\ de Melo $^{1 \bullet}$, Ycaro Kylldary Macêdo ${ }^{1}{ }^{\ominus}$, Maria Christina Sanches Muratori ${ }^{2}{ }^{\ominus}$, Julliet Teixeira \\ de Oliveira Santos ${ }^{30}$ \\ ${ }^{I}$ Estudante de Medicina Veterinária na Universidade Federal do Piauí, Departamento de Morfofisiológia Veterinária. Teresina -PI Brasil. \\ ${ }^{2}$ Professora Titular do departamento Morfofisiologia Veterinária Centro de Ciências Agrárias- UFPI \\ ${ }^{3}$ Mestre-Universidade federal do Piauí \\ *Autor para correspondência, E-mail:la_danielle@hotmail.com
}

\begin{abstract}
Resumo. A forma de acondicionamento de rações peletizadas pode influir diretamente na sua qualidade. Dessa forma, objetivou-se verificar a contaminação fúngica, em rações para cães vendidas a granel em diversos estabelecimentos da cidade de Teresina - PI. Foram avaliadas 15 amostras da mesma marca de cinco estabelecimentos comerciais de três regiões administrativas escolhidas aleatoriamente (A, B e C), de outubro a novembro de 2017. As regiões administrativas com maior contagem de fungos filamentosos e leveduras foi A seguida de C. Sendo o Aspergillus o gênero prevalente, presente em $71,4 \%$ das amostras analisadas.
\end{abstract}

Palavras-Chave: Alimentação animal, aspergillus, alternaria

\section{Prevalence of fungal contamination in rations sold in bulk in the city of Teresina, Piauí}

\begin{abstract}
The form of packaging of pelleted feed can directly influence its quality. Thus, the objective was to verify the fungal contamination in dog food sold in bulk in several establishments in the city of Teresina - PI. Fifteen samples of the same brand from five commercial establishments from three randomly selected administrative regions (A, B and C) were evaluated from October to November 2017. The administrative regions with the highest count of filamentous fungi and yeast was followed by C. Aspergillus is the prevalent genus present in $71.4 \%$ of the analyzed samples.
\end{abstract}

Keywords: Animal feed, aspergillus, alternaria

\section{Prevalencia de contaminación fúngica en raciones vendidas a granel en la ciudad de Teresina, Piauí}

Resumen. La forma de envasado de las raciones peletizadas puede influir directamente en su calidad. Por lo tanto, el objetivo fue verificar la contaminación por hongos en alimentos para perros vendidos a granel en varios establecimientos en la ciudad de Teresina - PI. Quince muestras de la misma marca de cinco establecimientos comerciales de tres regiones administrativas seleccionadas al azar (A, B y C) se evaluaron de octubre a noviembre de 2017. Las regiones administrativas con el mayor recuento de hongos filamentosos y levaduras fueron A seguida de C. Siendo el Aspergillus, el género prevalente, presente en el $71,4 \%$ de las muestras analizadas.

Palabras-clave: Alimentación animal, aspergillus, alternaría 


\section{Introdução}

Diante do aumento da criação de animais de companhia, o consumo de alimentos para cães e gatos cresceu bastante no Brasil, em que a preocupação maior tem sido sua saúde e bem-estar, sendo o principal motivo que tem levado ao consumidor buscar por uma ração industrializada de boa qualidade a qual contém uma fonte segura. As fábricas de ração têm inserido programas de autocontrole que visam à prevenção de contaminação pelo controle sobre o processo, obtendo melhora por parte do consumidor obtendo aumento sobre o lucro (Gazzotti et al., 2015).

O crescimento de fungos na ração animal é um problema em toda parte do mundo, que acarreta na perda da qualidade dos grãos, em decorrência principalmente da presença de micotoxinas que são biocontaminantes produzidos por alguns fungos toxigênicos, causando doenças e consequentemente mortes (Bennett \& Moore, 2015). Quando ocorre à multiplicação de bactérias e fungos na ração, há liberação de substâncias denominadas de exo-enzimas o que provoca mudanças nas propriedades dos alimentos e aumentando o risco de intoxicação (Bennett \& Moore, 2015; Bryden, 2012; Pereira et al., 2014).

Para o controle das micotoxinas causadas por fungos é necessária a realização de medidas preventivas no manejo dentre os quais se destacam a redução do período de armazenamento, manutenção dos teores de umidade e temperatura, redução na população de insetos nas plantações e umidades e de desempenho. O controle físico, sanitário ou nutricional da matéria prima é essencial, devendo ser criteriosamente observada a coloração, odor, presença de insetos, roedores e contaminantes, textura, uniformidade e temperatura de estocagem (Gazzotti et al., 2015; Zachariasova et al., 2014).

Este trabalho tem, portanto, o objetivo de verificar a contaminação fúngica, em rações para cães vendidas a granel em diversos estabelecimentos da cidade de Teresina - PI.

\section{Material e métodos}

As amostras de ração standards para cães foram adquiridas em três regiões administrativas ("A", "B" e "C") do município de Teresina, Piauí escolhidas ao acaso. Em cada região administrativa foram selecionados aleatoriamente cinco estabelecimentos e neles coletados amostras de $500 \mathrm{~g}$ de ração recolhidos diretamente de uma embalagem que estava aberta e exposta a venda no momento. O período total de coletas foi compreendido de outubro e novembro de 2017. As amostras foram adquiridas em embalagens de polietileno individuais, fornecidas pelo próprio estabelecimento e enviadas ao Laboratório de Controle Microbiológico de Alimentos, do Núcleo de Estudos e Pesquisa e Processamento de Alimentos da Universidade Federal do Piauí, para realização das posteriores análises micológicas.

A contagem de fungos filamentosos em unidades formadoras de colônias por grama de alimento (UFC. $\mathrm{g}^{-1}$ ) foi realizada segundo metodologia de diluição decimal seriada em placas descrita por Pitt \& Hocking (2009). Foram homogeneizados $25 \mathrm{~g}$ de cada amostra em $225 \mathrm{~mL}$ de água peptonada a $0,1 \%$ esterilizada. A partir dessa diluição inicial $\left(10^{-1}\right)$ foram preparadas diluições decimais seriadas até $10^{-3}$. A inoculação de cada uma das diluições foi efetuada em duplicata com alíquotas de $0,1 \mathrm{~mL}$ por placa de Petri, semeadas na superfície do meio de cultivo dichloran rose bengal cloranfenicol (DRBC) com auxílio de alça de Drigalski esterilizada (King et al., 1979). As placas com DRBC foram incubadas a $25^{\circ} \mathrm{C}$ durante sete dias em estufas microbiológicas com controle eletrônico de temperatura. Transcorrido tal período, todas as placas foram observadas, sendo selecionadas para contagem aquelas que continham entre 10 a 100 UFC. ${ }^{-1}$ (Dalcero et al., 1997; Dalcero et al., 1998).

A identificação de gênero de todas as colônias consideradas como diferentes foi realizada segundo Samson et al. (2000) de acordo com suas características macro e microscópicas. O delineamento experimental utilizado foi inteiramente ao acaso, com três tratamentos representados por regiões (A, B e C) e cinco repetições (estabelecimentos comerciais) perfazendo um total de 15 amostras. Os dados das contagens de fungos filamentosos e leveduras foram transformados em $\log ^{10}(x+1)$, realizou-se o teste de análise de variância para comparação das médias pelo pacote estatístico SigmaStat ${ }^{\circledR}$ v.4.0 com significância de 5,0\%. 


\section{Resultados e discussão}

As análises possibilitaram o isolamento de fungos pertencentes aos gêneros Aspergillus e Alternaria (Figura 1) em 100\% das amostras, com predominância de Aspergillus em 71,4\%. Tal achado também foi observado por Bueno et al. (2001), Andrade \& Nascimento (2005) e Barbosa et al. (2014) (Tabela 1). As amostras apresentaram nível de contaminação variando de 0,5 a 1,9 UFC/g, havendo diferença estatística entre a média da zona $\mathrm{C}$ e as zonas A e B (Tabela 2).

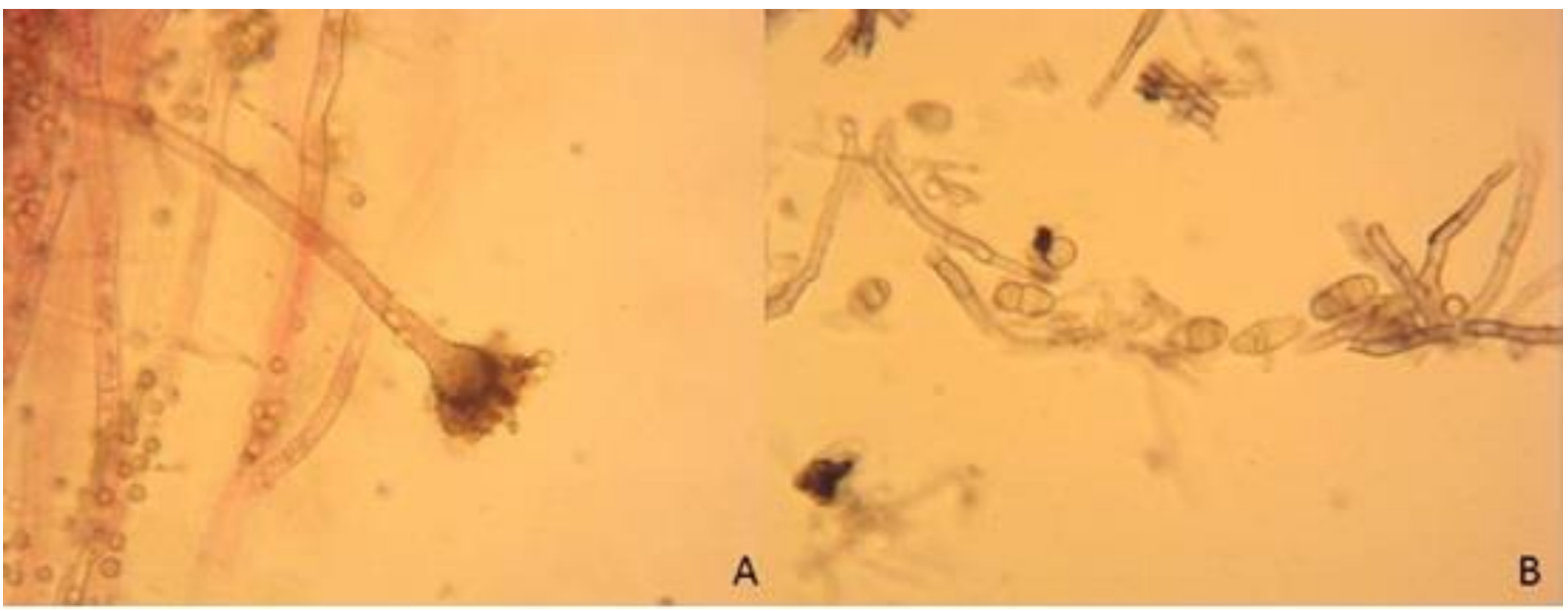

Figura 1. (A): Fungo do gênero Penicillium observado em isolamento fúngico proveniente de ração para cães. (B): Fungo do gênero alternaria observado em isolamento fúngico proveniente de ração para cães.

O pó que advém do solo pelo vento, chuva e retirada mecânica é a principal causa de contaminação vegetal dos grãos e sementes oleaginosas, podendo entrar em contato com os microrganismos durante o plantio, colheita, armazenamento e na transportação para o destino final (Pires et al., 2014).

Tabela 1 Número de isolados de gênero fúngico e ocorrência em amostras de ração para cães adquiridas em Teresina, PI.

\begin{tabular}{lcc}
\hline Gênero fúngico & Número de isolados & Ocorrência $(\%)$ \\
\hline Aspergillus spp. & 5 & 71,43 \\
Alternaria spp. & 2 & 28,57 \\
\hline Total & 7 & 100 \\
\hline
\end{tabular}

São classificados em três categorias os fatores para o crescimento de fungos e produção de toxinas: fatores químicos, físicos e biológicos. O armazenamento é um fator importante a ser levado em conta, o qual a recomendação mais indicada é manter o silo fechado não sendo permitido o contato com o ambiente externo, além de manter a temperatura e umidade controlada evitando a proliferação de fungos micotoxigênicos. A umidade é um grande fator para o desenvolvimento microbiano, sendo as partículas de pó contendo alta umidade as mais propensas para o crescimento de fungos e bactérias como, por exemplo, a Salmonella spp. (Forsythe, 2013; Franco \& Landgraf, 2003).

Tabela 2. Contagem de fungos filamentosos em rações para cães adquiridos em Teresina, PI.

\begin{tabular}{lc}
\hline Zona/Amostras & Contagem de fungos $\left(\mathrm{UFC}_{\mathrm{g}}{ }^{-1} \mathrm{em}^{\log } \mathrm{o}^{(\mathrm{x}+1)}\right)$ \\
\hline A & $1,9^{\mathrm{a}} \pm 0,7$ \\
B & $1,6^{\mathrm{a}} \pm 0,5$ \\
C & $0,5^{\mathrm{b}} \pm 0,5$ \\
\hline
\end{tabular}

${ }^{a}$ letras iguais em linhas, resultados semelhantes $(\mathrm{P}>0,05)$ pelo teste de Tukey. UFC. $\mathrm{g}^{-1} \mathrm{em} \log _{10}{ }^{(\mathrm{x}+1)}=$ unidades formadoras de colônias por grama, em logaritmos da base dez, acrescentados de uma unidade.

As micotoxinas são agentes químicos produzidos a partir do metabolismo secundário de fungos filamentosos contaminando alimento dos animais promovendo efeitos agudos ou crônicos. Aflatoxinas são toxinas produzidas por fungos comumente encontradas em rações infectadas especialmente em áreas com clima quente e úmido, sendo uma micotoxina que pode causar maior intoxicação para saúde animal devido sua ampla ocorrência e toxicidade. Há vários tipos de aflatoxinas produzidas, dentre as quais 
podem ser citadas a Aflatoxina B1, Aflatoxina B2, Aflatoxina G1 e Aflatoxina G2, sendo o principal fungo do gênero Aspergillus o mais comum (Midio \& Martins, 2000).

Apesar das estratégias utilizadas pelas fábricas de ração para o combate à contaminação fúngica em seus produtos, ainda assim é possível detectar o crescimento desses microrganismos. A evidenciação de fungos do gênero Penicillium é preocupante devido à capacidade toxigênica da espécie (Pires et al., 2014). Há necessidade de cuidado com o local de armazenamento, recipientes e containers do produto final, sendo verificada também a capacitação dos funcionários e conscientização dos mesmos para o sucesso no controle biológico. Todos os ingredientes que constituem a produção de alimentos devem ser registrados pelo MAPA, não devendo ser aceito aqueles que contenham parasitas, substâncias toxicas ou microrganismos. O produto final deve atender os padrões de identidade e qualidade específicas para cada produto (Andrade \& Nascimento, 2005).

A ocorrência de nível de contaminação superior deve-se provavelmente à origem das amostras analisadas que foram adquiridas na modalidade a' granel, as quais ficam expostas às condições de temperatura, pressão, contato com as demais rações e manuseio constante dos vendedores e clientes para retirada, o que incrementa a possibilidade de crescimento microbiano. O contato com as partículas presentes no ar é a principal causa de contaminações em produtos de origem vegetal, o que corresponde a um dos maiores componentes das rações avaliadas na literatura (Barbosa et al., 2014; Cappelli et al., 2016).

A diferença estatística obtida entre as médias de contagem dos fungos da zona $\mathrm{C}$ e as zonas A e B pelo teste de Tukey a $5 \%$ de significância ocorreram provavelmente devido à localização dos estabelecimentos. Apenas a média de contagem da zona $\mathrm{C}$ diferiu dentre as demais pelo teste de Tukey a 5\%, por se localizar em área mais movimentada da cidade, pode apresentar maior índice de vendas, ocasionando uma troca mais frequente do material comercializado, diminuindo o tempo em prateleira, possibilitando menor tempo de contato com o ar, portanto, menores níveis de contaminação embora ainda existente (Bennett \& Moore, 2015; Kumar et al., 2008; Magan \& Aldred, 2007).

As rações para os cães podem ser armazenadas em embalagem fechada e a granel, sendo está a opção mais barata para o consumidor. Diante disso, vale ressaltar o perigo da venda à granel, visto que há maior risco de contaminação por microrganismos como os fungos, devido ao contato direto com o ar (Cappelli et al., 2016).

\section{Conclusão}

As rações para os cães devem ser armazenadas de preferência em embalagem fechada, havendo o risco de contaminação para os animais domésticos de micotoxinas presentes na ração oriundas de fungos. Pode-se observar que as micotoxinas mais encontradas nos alimentos são as aflatoxinas sendo que a gravidade está relacionada com a toxicidade presente.

\section{Referências bibliográficas}

Andrade, R. M. \& Nascimento, J. S. (2005). Presença de fungos filamentosos em ração para cães comercializadas na cidade de Pelotas-RS. Arquivo do Instituto Biológico de São Paulo, 7110-12.

Barbosa, I. P., Rodrigues, A. M. D., Muratori, M. C. S., Ferreira, M. D. S., Cardoso Filho, F. C. \& Pereira, M. M. G. (2014). Espécies fúngicas isoladas de ração para gatos comercializadas. PUBVET, 81822-1939.

Bennett, J. W. \& Moore, G. G. (2015). Mycotoxins. Reference Module in Biomedical Sciences, 16497516.

Bryden, W. L. (2012). Mycotoxin contamination of the feed supply chain: Implications for animal productivity and feed security. Animal Feed Science and Technology, 173(1):134-158.

Bueno, D. J., Silva, J. O. \& Oliver, G. (2001). Mycoflora in commercial pet foods. Journal of Food Protection, 64(5):741-743.

Cappelli, S., Lunedo, P., Freitas, C. P., Raber, H. R., Manica, E., Hashimoto, J. H. \& Oliveira, V. (2016). Avaliação química e microbiológica das rações secas para cães e gatos adultos comercializadas a granel. Revista Brasileira de Higiene e Sanidade Animal, 10(1):90-102. 
Dalcero, A., Magnoli, C., Chiacchiera, S., Palacios, G. \& Reynoso, M. (1997). Mycoflora and incidence of aflatoxin B 1, zearalenone and deoxynivalenol in poultry feeds in Argentina. Mycopathologia, 137(3):179-184.

Dalcero, A., Magnoli, C., Luna, M., Ancasi, G., Reynoso, M. M., Chiacchiera, S., . . Palacio, G. (1998). Mycoflora and naturally occurring mycotoxins in poultry feeds in Argentina. Mycopathologia, 141(1):37-43.

Forsythe, S. J. (2013). Microbiologia da segurança dos alimentos. Porto Alegre, Rio Grande do Sul, Brasil: Artmed.

Franco, B. D. G. M. \& Landgraf, M. (2003). Microbiologia dos alimentos. São Paulo: Atheneu.

Gazzotti, T., Biagi, G., Pagliuca, G., Pinna, C., Scardilli, M., Grandi, M. \& Zaghini, G. (2015). Occurrence of mycotoxins in extruded commercial dog food. Animal Feed Science and Technology, 20281-89.

King, A. D., Hocking, A. D. \& Pitt, J. I. (1979). Dichloran-rose bengal medium for enumeration and isolation of molds from foods. Applied and Environmenatal Microbiology, 37(5):959-964.

Kumar, V., Basu, M. S. \& Rajendran, T. P. (2008). Mycotoxin research and mycoflora in some commercially important agricultural commodities. Crop Protection, 27(6):891-905.

Magan, N. \& Aldred, D. (2007). Post-harvest control strategies: Minimizing mycotoxins in the food chain. International Journal of Food Microbiology, 119(1-2):131-139. doi: http://dx.doi.org/10.1016/j.ijfoodmicro.2007.07.034

Midio, A. F. \& Martins, D. I. (2000). Toxicologia de Alimentos. São Paulo, Brasil: Varela Editora e Livraria Ltda.

Pereira, V. L., Fernandes, J. O. \& Cunha, S. C. (2014). Mycotoxins in cereals and related foodstuffs: A review on occurrence and recent methods of analysis. Trends in Food Science \& Technology, 36(2):96-136.

Pires, P. G. S., Teixeira, L. \& Mendes, J. V. (2014). Composição nutricional e avaliação de rótulos de rações secas para cães e gatos adultos comercializadas em Pelotas - RS. . Enciclopédia Biosfera, 10(18):1001-1008.

Pitt, J. I. \& Hocking, A. D. (2009). Fungi and food spoilage. London, UK.: Springer.

Samson, R. A., Hoekstra, E. S., Frisvad, J. C. \& Filtenborg, O. (2000). Introdution to food-borne fungi. Berlin: Centralalbureau voor Schimmelcultures Baarn Deft.

Zachariasova, M., Dzuman, Z., Veprikova, Z., Hajkova, K., Jiru, M., Vaclavikova, M., . . Hajslova, J. (2014). Occurrence of multiple mycotoxins in European feedingstuffs, assessment of dietary intake by farm animals. Animal Feed Science and Technology, 193124-140.

Recebido: 23 de setembro, 2019.

Aprovado: 14 de novembro, 2019.

Publicado: 3 de janeiro, 2020.

Licenciamento: Este artigo é publicado na modalidade Acesso Aberto sob a licença Creative Commons Atribuição 4.0 (CC-BY 4.0), a qual permite uso irrestrito, distribuição, reprodução em qualquer meio, desde que o autor e a fonte sejam devidamente creditados. 